\title{
MicroRNAs 143 and 145 are possible common onco-microRNAs in human cancers
}

\author{
YUKIHIRO AKAO $^{1}$, YOSHIHITO NAKAGAWA ${ }^{1}$ and TOMOKI NAOE ${ }^{2}$ \\ ${ }^{1}$ Gifu International Institute of Biotechnology, 1-1 Naka-Fudogaoka, Kakamigahara, Gifu 504-0838; ${ }^{2}$ Department of \\ Hematology, Nagoya University, Graduate School of Medicine, 65 Tsurumai-cho, Showa-ku, Nagoya 466-8550, Japan
}

Received May 23, 2006; Accepted June 23, 2006

\begin{abstract}
MicroRNAs (miRNAs) are endogenously expressed RNAs 18-24 nucleotides in length that regulate gene expression through translational repression by binding to a target mRNA. It is thought that the expression level of miRNAs, which act like antioncogenes, is frequently reduced in cancers because of chromosome deletion and epigenetic changes. Since the processing of miRNAs has been characterized to be enzymatic in nature, the expression levels of miRNAs are closely associated with the activity and levels of such enzymes. Here, we found that miRNA 143 and 145 expression levels were extremely reduced in colon cancer cells and commonly in the other kinds of cancer cells tested. The transfection of each precursor miRNA into the cells demonstrated a significant growth inhibition in human colon cancer DLD-1 and SW480 cells, and ERK5 was determined to be the target gene of miRNA 143. Since the presence of genomic loci of the miRNAs was confirmed by PCR in the cell lines and the precursor miRNAs exhibited a growth inhibitory effect in DLD-1 and SW480 cells, the early processes such as transcription and enzymatic modification from primary miRNAs to precursor miRNAs seemed to be commonly disturbed. These findings indicate that the miRNAs 143 and 145 could become good tumor markers and provide an important clue in the study of the mechanism of oncogenesis involving miRNAs.
\end{abstract}

\section{Introduction}

MicroRNAs (miRNAs) are a recently discovered class of small non-coding transcripts that regulate gene expression by promoting the degradation of transcribed mRNAs or by inhibiting their translation (1). Although bioinformatic approaches suggest that miRNAs may regulate the expression of a large fraction of the genome $(2,3)$, the determination of

Correspondence to: Dr Yukihiro Akao, Gifu International Institute of Biotechnology, 1-1 Naka-Fudogaoka, Kakamigahara, Gifu 504-0838, Japan

E-mail: yakao@giib.or.jp

Key words: microRNAs 143 and 145, human cancer, oncomiRNA, ERK5, growth inhibition
miRNA gene targets and biological fractions has been comparatively limited. More than 400 miRNAs have been identified in the human (miR registry, www.sanger.ac. uk/Software/Rfam/mirna/index.shtml). Emerging studies suggest that many miRNAs may participate in human disease, especially in the case of oncogenesis $(4,5)$. Changes in the levels of miRNAs may alter the control of growth or apoptosis in some cancers (6-8). A reduction in the level of miRNAs has been reported to occur in various cancers $(9,10)$. Chromosomal aberrations such as mutation, amplification, deletion and translocation, as well as epigenetic changes, are frequently observed in cancers, which cause not only deregulation of mRNA expression, but also inappropriate miRNA expression. There are some reports that the level of let-7 was decreased in lung tumors and this findings predicted the prognosis of patients (9). It was also reported that let-7 miRNA negatively regulated the oncogenes RAS and c-myc at the translational level (11). In such situations, we have sought to clarify whether miRNAs are truly associated with the cell proliferation and differentiation, and what oncogenes are regulated at the translational level by the miRNAs. Here, we found that miRNA (mir)s 143 and 145 were associated with the oncogenesis by showing a decrease in their expression levels. This association was commonly observed in the various cancers tested. These findings afford the possibility that these miRNAs may become tumor markers, and a target of study to learn how the miRNAs contribute to carcinogenesis.

\section{Materials and methods}

Patients and tissue preparation. All human tissue samples were specimens from patients who had undergone biopsy for histological diagnosis of colon cancer at Saiseikai Ibaraki hospital, Ibaraki, Osaka, between 2000 and 2003. Informed consent in writing was obtained from each patient. The tumor and its adjacent normal tissues were obtained. Each specimen was divided into 2 parts. One was subjected to Western blot analysis, and the other was used for extraction of RNA. The total-RNAs from human normal tissues were purchased from BioChain Institute, Inc. (Hayward, CA).

DNA array hybridization. Fractions containing RNA species smaller than $30 \mathrm{nt}$ were isolated from total-RNA samples by a rapid column gel-electrophoresis method (Ambion Inc., Austin, TX). miRNAs were 3 ' labeled with poly(A) poly- 
Table I. Summary of clinicopathologic features in human colorectal tumors.

\begin{tabular}{lclrlll}
\hline Case no. & Age & Sex & Size $(\mathrm{mm})$ & \multicolumn{1}{c}{ Site } & Pathological findings (differentiation) & Dukes' stage \\
\hline 1 & 44 & Female & $55 \times 25 \times 15$ & Sigmoid & Well & C \\
2 & 62 & Female & $67 \times 55 \times 20$ & Ascending & Well & C \\
3 & 50 & Male & $120 \times 74 \times 25$ & Rectum & Well & Moderate \\
4 & 65 & Male & $50 \times 40 \times 15$ & Rectum & Moderate & C \\
5 & 62 & Female & $60 \times 60 \times 25$ & Caecum & Well & C \\
6 & 70 & Male & $70 \times 60 \times 28$ & Sigmoid & C \\
\hline
\end{tabular}

merase, amino-allyl UTP, and Cy-3 or Cy-5 dye. Labeled miRNAs were hybridized on glass-slide microarrays carrying DNA oligonucleotide probes specific for each known miRNA.

Cell culture and cell viability. Human malignant cell lines SW480, DLD-1 and COLO101 (colon cancer); HL60 (myeloid leukemia); U937 (monocytic leukemia); Jurkat (T-cell leukemia); HeLa (uterine cervix); HuH7 (hepatic cancer); PC3 and LNCaP (prostate cancer); and SHSY-5Y (neuroblastoma) were grown in RPMI-1640 medium supplemented with $10 \%$ (v/v) heat-inactivated FBS (Sigma, St. Louis, MO, USA) and $2 \mathrm{mM}$ L-glutamine under an atmosphere of $95 \%$ air and $5 \%$ $\mathrm{CO}_{2}$ at $37^{\circ} \mathrm{C}$. The number of viable cells was determined by the trypan-blue dye exclusion test. 5-Aza-2'-deoxycytidine (Sigma) was used for demethylation of DNA and histone.

Semi-quantitative RT-PCR and genomic PCR. Total-RNA was isolated from the cells and tissues by the phenol/guanidium thiocyanate method with DNase I treatment. To determine the expression of miRNAs, we measured their levels by using a mirVana ${ }^{\mathrm{TM}}$ qRT-PCR miRNA detection kit (Ambion) and mirVana $^{\mathrm{TM}}$ qRT-PCR Primer set (Ambion). Briefly, after reverse transcription of $50 \mathrm{ng}$ of total-RNA, cDNA was generated. The PCR reaction consisted of 22 cycles $\left(95^{\circ} \mathrm{C}\right.$ for $15 \mathrm{sec}, 60^{\circ} \mathrm{C}$ for $\left.30 \mathrm{sec}\right)$ after an initial denaturation step $\left(95^{\circ} \mathrm{C}\right.$ for $3 \mathrm{~min}$ ). U6 was used as an internal control. The PCR primer pairs for mirs-143 and -145 were obtained commercially from Ambion. The PCR products by using such primer pairs were confirmed to be from loci of mirs-143 and -145 by DNA sequencing.

To determine the levels of mRNAs, we purified prepared cDNA samples by use of a PCR purification kit (Qiagen, Hilden, Germany) and used them for PCR (Takara, Ohtsu, Japan). The primers for ERK5 were as follow: ERK5-sense211, 5'-CCTTCGATGTGACCTTTGAC-3'; and ERK5-antisense-1418, 5'-TGACACCATTGATCTGACCC-3'.

To examine the presence of the genomic loci of mirs-143 and -145 , we extracted DNA from the cell lines tested and used it for PCR (Takara). The primers for genomic loci of mirs-143 and -145 were as follow: 5q32-sense, 5'-TTGGTCC TGGGTGCTCAAAT-3'; and 5q32-antisense, 5'-AGGAACT CCCAAGCTCAAGT-3'. The primers amplified the DNA fragment including the both loci at 5q32. The genomic locus of glyceraldehyde phosphate dehydrogenase (GAPDH) was used as an internal control. The PCR reaction consisted of 30 cycles $\left(94^{\circ} \mathrm{C}\right.$ for $30 \mathrm{sec}, 57.5^{\circ} \mathrm{C}$ for $1 \mathrm{~min}, 72^{\circ} \mathrm{C}$ for $1 \mathrm{~min}$ ) after an initial denaturation step $\left(95^{\circ} \mathrm{C}\right.$ for $\left.1 \mathrm{~min}\right)$. The PCR products were analyzed by electrophoresis on $2 \%$ agarose gels.

Transfection of DLD-1 and SW480 cells with mir-143 or-145 precursor miRNAs. DLD-1 and SW480 cells were seeded in 6 -well plates at a concentration of $1-2 \times 10^{5} /$ well $(30-40 \%$ confluence) the day before the transfection. The mirs-143 and -145 precursor miRNAs (Ambion) were used for the transfection of the cells $(20-80 \mathrm{nM} / \mathrm{ml})$, which was achieved by using cationic liposomes TransIT-TKO (Mirus Bio Co., Madison, WI) according to the manufacturer's Lipofection protocol. The transfection efficiency was evaluated by the transfection of the cells with a duplex siRNA-FITC (Dharmacon, Lafayette, CO). Non-specific control miRNA (NS, 57\% GC content; Ambion) was used as a control for non-specific effects. The precursor mirs-143 and -145 were UGAGAUGAAGCACUGUAGCUCA and GUCCAGUUU UCCCAGGAAUCCCUU, respectively. The effects manifested by the introduction of mir-143 or -145 precursor miRNAs into the cells was assayed at $48 \mathrm{~h}$ after the transfection by Western blot and qRT-PCR analyses for mRNA and miRNA.

Western blotting. The cells were homogenized in chilled lysis buffer comprising $10 \mathrm{mM}$ Tris- $\mathrm{HCl}$ (pH 7.4), 1\% NP-40, $0.1 \%$ deoxycholic acid, $0.1 \%$ SDS, $150 \mathrm{mM} \mathrm{NaCl}, 1 \mathrm{mM}$ EDTA and $1 \%$ Protease Inhibitor Cocktail (Sigma) and stood for $30 \mathrm{~min}$ on ice. After centrifugation at 13,000 rpm for $20 \mathrm{~min}$ at $4^{\circ} \mathrm{C}$, the supernatants were collected as protein samples. Protein contents were measured with a DC Protein assay kit (BioRad, Hercules, CA, USA). Ten micrograms of lysate protein for Western blotting of ERK5 and c-myc was separated by SDS-PAGE using a $10 \%$ polyacrylamide gel and electroblotted onto a PVDF membrane (Du Pont, Boston, MA). After blockage of non-specific binding sites for $1 \mathrm{~h}$ with $5 \%$ non-fat milk in PBS containing $0.1 \%$ Tween-20, the membrane was incubated overnight at $4^{\circ} \mathrm{C}$ with anti-human ERK5 antibody (Cell Signaling Tec. Inc., Berverly, MA) or with antihuman c-myc antibody (Santa Cruz, CA). The membranes were then washed 3 times with PBS containing 0.1\% Tween-20, incubated further with HRP-conjugated sheep anti-mouse or donkey anti-rabbit Ig antibody (Amersham Biosciences, Piscataway, NJ) at room temperature, and then washed 3 times with PBS containing $0.1 \%$ Tween-20. The immunoblots were visualized by use of an enhanced chemiluminescence detection kit (New England Biolabs, Beverly, MA, USA). 


\section{Human colorectal tumors

\begin{tabular}{|c|c|c|c|c|c|c|c|c|c|c|c|c|}
\hline \multicolumn{2}{|c|}{1} & \multicolumn{2}{|c|}{2} & \multicolumn{2}{|c|}{3} & \multicolumn{2}{|c|}{4} & \multicolumn{2}{|c|}{5} & \multicolumn{2}{|c|}{6} & \\
\hline$T$ & $\mathbf{N}$ & $T$ & $\mathbf{N}$ & $\mathbf{T}$ & $\mathbf{N}$ & $\mathbf{T}$ & $\mathbf{N}$ & $\mathbf{T}$ & $\mathbf{N}$ & $\mathbf{T}$ & $\mathbf{N}$ & \\
\hline$=$ & -8 & $=8$ & c & -8 & $\infty$ & 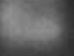 & 08 & $\mathrm{sin}$ & $-\infty$ & 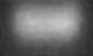 & $\infty$ & mir-143 \\
\hline$=$ & $=$ & $\infty$ & -8 & 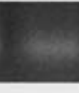 & $-\infty$ & 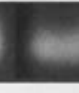 & $1=$ & E & 8 & E & 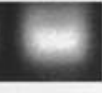 & mir-145 \\
\hline & & & & & & & & & & & & U6 \\
\hline
\end{tabular}

Figure 1. Expression of mirs-143 and -145 in human colon tumor tissues evaluated by qRT-PCR. The two pairs of lanes, $\mathrm{T}$ and N, contained samples from tumor tissues and the adjacent non-tumor tissues, respectively. The patient number corresponds to that in Table I. U6 was used as an internal standard.

\section{Normal human tissues}

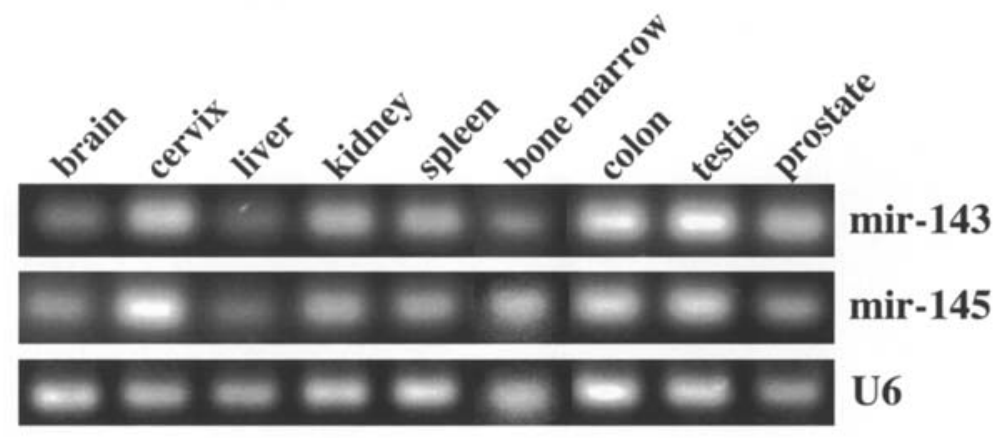

Human malignant cell lines

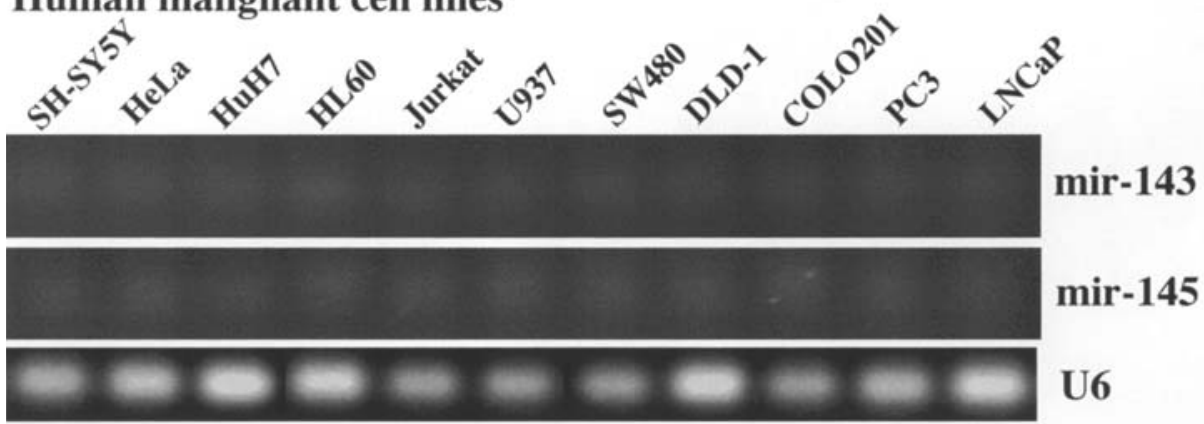

Figure 2. qRT-PCR-evaluated expression of mirs-143 and -145 in various human cancer cell lines. The expression of the miRNAs in normal human tissues (upper panel) and in various cancer cell lines (lower panel) is shown. U6 was used as an internal standard.

\section{Results}

We examined the expression profile of miRNAs from DLD-1 cells by the hybridization of the miRNAs to the DNA microarrays for miRNAs (data not shown). From the results of DNA array hybridization, we focused on the mirs-143 and -145 among the low-expression miRNAs in the experiment. In order to determine the expression levels of mirs-143 and -145 , we examined the expression of both miRNAs in the colon tumor samples (Table I) by qRT-PCR. As shown in Fig. 1, in 5 of 6 patients the expression levels of both miRNAs in tumors were significantly decreased compared with those in the paired normal samples, which is almost the same results as obtained by Michael et al (10). Furthermore, we examined the expression levels of the miRNAs in various kinds of human cancer cell lines. Interestingly, all the cell lines tested showed a considerably low expression of mirs-143 and -145 , whereas the expression in the human normal tissue from which each cell line had originated was fairly good (Fig. 2). Among the human tissues tested, uterine cervix, colon and testis expressed a greater amount of mirs-143 and -145 than the other tissues. The pattern of the expression of both was very similar in the human normal tissues (upper panel).

To validate the function of both miRNAs in cell growth, we next used the mir-143 or -145 precursor miRNAs for transfection of the human colon cancer DLD-1 and SW480 cells, both of which showed a good transfection efficiency by the use of FITC-siRNAs (data not shown). After the transfection, the levels of both miRNAs were increased, which indicated that the enhancement was due to the introduction of each precursor miRNA into the cells (Fig. 3A). The cell growth of both cell 
(A)

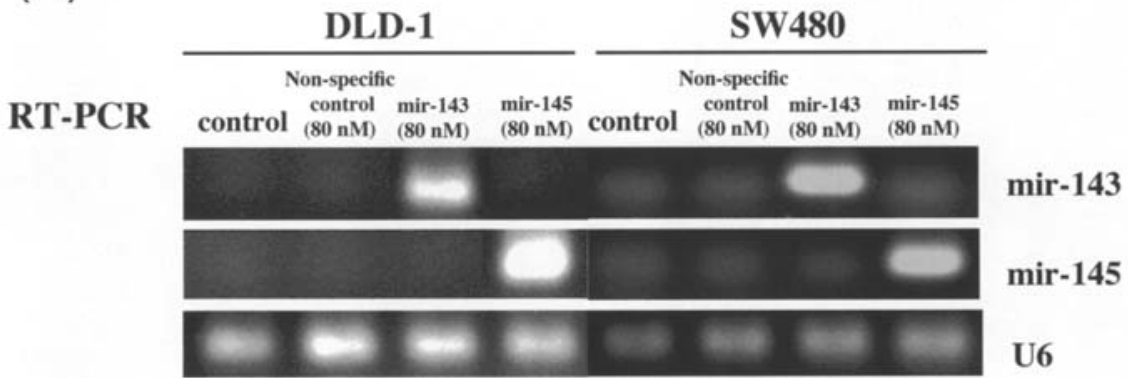

(B)
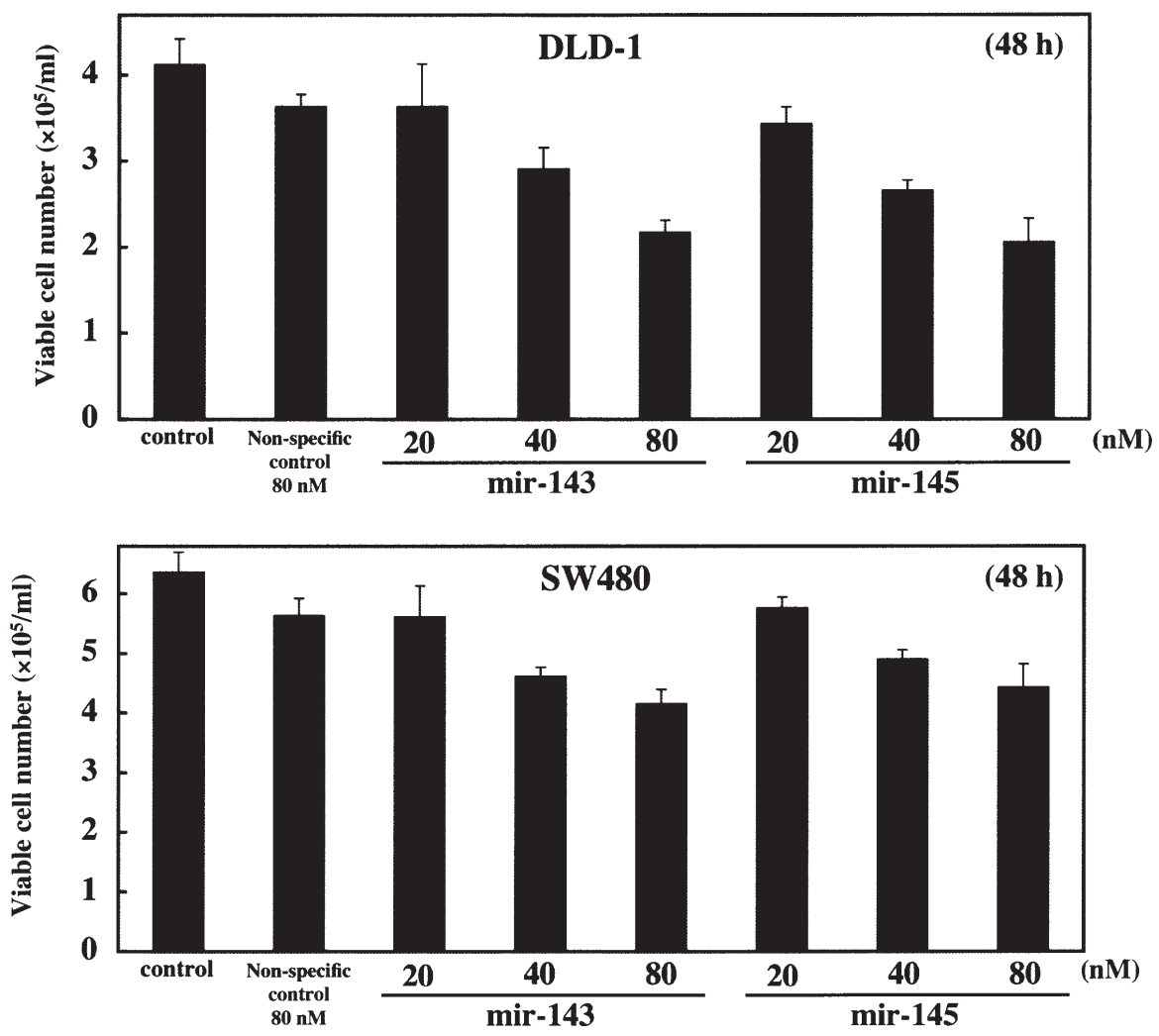

C)

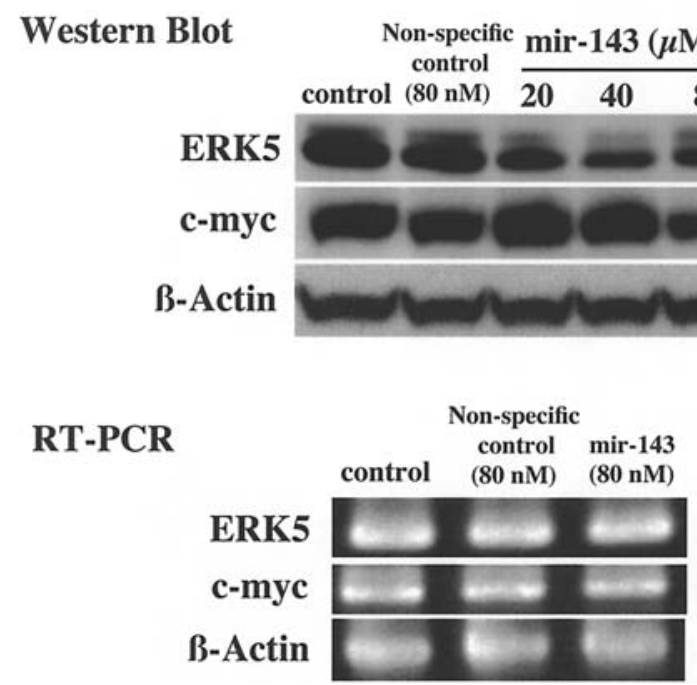

Figure 3. Effect of transfection of human colon cancer DLD-1 and SW480 cells with mir-143 or -145 precursor miRNAs. (A), Levels of mirs-143 and -145 in DLD-1 and SW480 cells after the transfection of the cells with mir-143 or -145 precursor miRNAs at $80 \mathrm{nM}$. (B), Number of viable transfected or control cells at $48 \mathrm{~h}$ after transfection, which evaluation was performed at $24 \mathrm{~h}$ after inoculating the cells into the culture dishes (1x105/ml). Data represent the mean $\pm \mathrm{SD}$ of 3 different experiments, each carried out in duplicate. (C), Expression levels of ERK 5 and c-myc proteins and of ERK5 and $c$-myc mRNAs at 48 h after the transfection of DLD-1 cells with mir-143 precursor miRNAs, as evaluated by Western blot analysis (upper panel) and by qRT-PCR (lower panel), respectively, at $48 \mathrm{~h}$ after the transfection of the cells. $\beta$-actin was ued as an internal standard. Control cells were incubated in medium containing transfection reagent alone. 


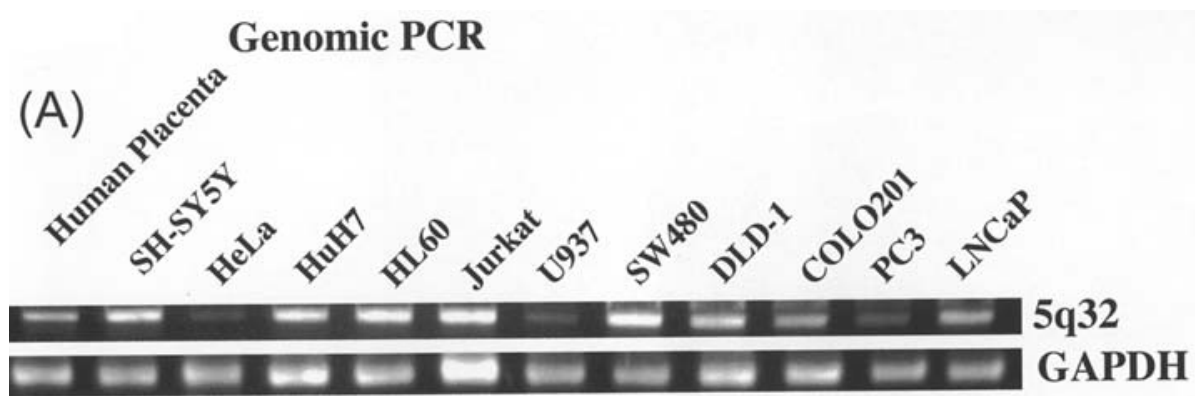

B)

\section{qRT-PCR}

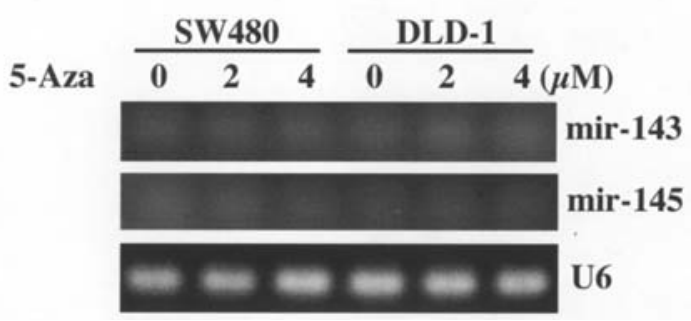

Figure 4. Confirmation of the presence of the genomic loci of mirs-143 and -145 at chromosome $5 q 32$ (A) and the expression of the miRNAs in DLD-1 and SW480 cells after their treatment with 5-Aza-2'-deoxycytidine for $18 \mathrm{~h}$ (B). (A), The primers amplified the DNA fragment including the loci of mirs-143 and -145. The genomic locus of glyceraldehyde phosphate dehydrogenase (GAPDH) was used as an internal control. (B), The expression of the miRNAs in DLD-1 and SW480 cells after the treatment with 5-Aza-2'-deoxycytidine at the indicated concentrations for $18 \mathrm{~h}$ is shown. U6 was used as an internal standard. lines was significantly inhibited in dose-dependent manner at $48 \mathrm{~h}$ after the transfection with either mir-143 or -145 (Fig. 3B). The growth suppressive effects of both precursor miRNAs were almost even, but the effects of both on DLD-1 cells were higher than those on SW480 cells. It has been reported that mir-143 represses the expression of ERK5 translationally (12). Therefore, by Western blot analysis we examined the time course of the expression of ERK5 protein after the transfection. The results indicated that the level of the protein in DLD-1 cells was significantly reduced, in a dose-dependent manner, by the transfection with mir-143 precursor miRNAs (Fig. 3C). On the other hand, the levels of ERK5 mRNAs evaluated by qRT-PCR in DLD-1 cells were almost unchanged in the same samples (Fig. 3C; upper panel), which indicated that the exogenously transfected mir-143 precursor miRNAs caused the decreased expression of the ERK5 protein at the translational level. The level of c-myc protein, a downstream signal protein of ERK5 $(13,14)$, was also reduced after the transfection (Fig. 3C; lower panel).

In order to disclose which process of miRNAs was perturbed in human cancer cell lines, we searched for the genomic locations of mir-143 and -145 (miR registry, www. sanger.ac.uk/Software/Rfam/mirna/index.shtml). Surprisingly, both genomic loci were co-located within $2.0 \mathrm{~kb}$ at chromosome 5q32 (15). Therefore, we constructed the PCR primers to confirm the presence of the genomic region including both loci in the cell lines by PCR. As shown in Fig. 4A, the PCR products using the primer pair were detected in all the cell lines tested. However, in HeLa, U937 and PC3 cells, one allele of the loci may be deleted. To exclude the possibility that the DNA of the loci and the histone were methylated, we examined by qRT-PCR the expression of both miRNAs after treatment of the DLD-1 and SW480 cells with 5-Aza-2'-deoxycytidine at various concentrations. The levels of both miRNAs were found to be almost unchanged (Fig. 4B).

\section{Discussion}

We found that the expression levels of mirs-143 and -145 were reduced in colorectal tumor tissues and further that their expression was commonly decreased in the other types of human cancer cell lines tested. Both miRNAs are possible onco-miRNA markers, and it is certain that the clarification of the mechanisms responsible for the lowering of their expression levels will be a clue to aid our understanding of oncogenesis from a different stand-point. Furthermore, we demonstrated growth inhibition of DLD-1 and SW480 cells after the introduction of mir-143 or -145 precursor miRNAs into them, thus indicating that mir-143 and -145 contributed negatively to cell growth and suggesting the possibility of using these miRNAs as gene therapy for cancers. Micheal et al reported that mirs-143 and -145 consistently display reduced steady-state levels of the mature miRNA at the adenomatous and cancer stages of colorectal cancers and that in cancer cells decreased levels of mature miRNAs was due to reduced Dicer-processing activity. In our study, since the introduction of mir-143 or -145 precursors into the DLD-1 and SW480 cells caused growth inhibition, the activity and expression of Dicer and RNA-induced silencing complex (RISC) proteins which exert the enzymatic modification of precursor miRNAs seemed to be intact (16-18). Therefore, we propose that the cause of inadequate expression of mirs-143 and -145 was due to the perturbation of transcription and/or the enzymatic process of the transition from primary miRNAs to precursor ones including Drosha and DGCR8, events that occur in the nucleus $(16,17)$. We also confirmed that both miRNAs were decreased in all the human cancer cell lines tested such as brain, liver, cervical, prostate cancers and myelocytic leukemia as well as colon cancer by comparing with matched normal tissues.

Interestingly, the DNA loci of both miRNAs are very close to each other within approximate $2.0 \mathrm{~kb}$, which led us to speculate that both precursors originate from the same primary miRNA. Therefore, we considered that genomic deletions and epigenetic changes may have caused their low expression in the cell lines tested. However, the presence of the DNA loci was confirmed by genomic PCR and the results of qRT-PCR analysis of the miRNAs from the cells treated with 5-Aza-deoxycytidine indicated no methylation of DNA and/or histone of the loci. Further detailed cytogenetic study 
will be needed to understand the genomic deletion of the loci.

As to the target oncogene(s) of mir-143 or -145 , the predicted proteins targeted by mir-143 or -145 shown in the website of Sanger are more than 250 mRNAs. It has been reported that mir-143 negatively regulates ERK5 mRNAs at the translational level (12). We also obtained a similar result from the transfection experiments using mir-143 precursor miRNAs. ERK5 is known to promote cell growth and proliferation in response to tyrosine kinase $(13,14)$, therefore persistent dereased levels of mir-143 in cancer cells may be directly involved in carcinogenesis through activation of the mitogen-activated protein kinase (MAPK) cascade via ERK5. According to the target proteins of mir-145, although we searched for its several candidate proteins by Western blot, we could not determine it. However, MAPK transduction proteins such as MAP3K and MAP4K4 are possible targets for mir-145. Thus, in the case of the signal molecules in the MAPK system and transcription factors targeted by such an miRNA, the expression of many proteins would be affected, leading to deregulation of the signal transduction of cell growth, survival and death. $c-m y c$ is also lowered by the transfection of cells with mir-143 precursor miRNAs, perhaps due to the decreased expression of ERK5, because c-myc lies downstream of the cell growth signaling pathway including ERK5 (13,14).

A primary miRNA from $5 q 32$ probably generates both of the mature miRNAs that effect growth inhibition, and thereby same transcription factors could be involved in the transcription. Recently, Fazi et al have reported the gene reprogramming through transcription of mir-223 mediating two transcription factors NFI-A and $\mathrm{C} / \mathrm{EBP} \alpha$ in human granulocyte differentiation (19), which may be also a possible scenario in this case.

Further study to clarify the details of the machinery involved in miRNA processing and the association between the perturbation of RNA modification and carcinogenesis will be needed for a better understanding of the carcinogenesis involving miRNAs.

\section{Acknowledgements}

This study was supported by a Grant-in-Aid for scientific research from the Ministry of Education, Science, Sports and Culture of Japan.

\section{References}

1. Ambros V: MicroRNA pathways in flies and worms: growth, death, fat, stress and timing. Cell 113: 673-676, 2003.

2. Ambros V: The functions of animal microRNAs. Nature 431: 350-355, 2004.
3. Bartel DP: MicroRNAs: genomics, biogenesis, mechanism and function. Cell 116: 281-297, 2004.

4. McManus MT: MicroRNAs and cancer. Semin Cancer Biol 13: 253-258, 2003.

5. Croce CM and Calin GA: miRNAs, cancer and stem cell division. Cell 122: 6-7, 2005.

6. Hariharan M, Scaria V, Pillai B and Brahmachari SK: Targets for human encoded microRNAs in HIV genes. Biochem Biophys Res Commun 337: 1214-1218, 2005.

7. Alvarez-Garcia I and Miska EA: MicroRNA functions in animal development and human disease. Development 132: 4653-4662, 2005.

8. Cimmino A, Calin GA, Fabbri M, Iorio MV, Ferracin M, Shimizu M, Wojcik SE, Aqeilan RI, Zupo S, Dono M, Rassenti L, Alder H, Volinia S, Liu CG, Kipps TJ, Negrini M and Croce CM: miR-15 and miR-16 induce apoptosis by targeting BCL2. Proc Natl Acad Sci USA 102: 13944-13949, 2005.

9. Takamizawa J, Konishi H, Yanagisawa K, Tomida S, Osada H, Endoh H, Harano T, Yatabe Y, Nagino M, Nimura Y, Mitsudomi T and Takahashi T: Reduced expression of the let-7 microRNAs in human lung cancers in association with shortened postoperative survival. Cancer Res 64: 3753-3756, 2004.

10. Michael MZ, O'Connor SM, van Holst Pellekaan NG, Young GP and James RJ: Reduced accumulation of specific microRNAs in colorectal neoplasia. Mol Cancer Res 1: 882-891, 2003.

11. Johnson SM, Grosshans H, Shingara J, Byrom M, Jarvis R, Cheng A, Labourier E, Reinert KL, Brown D and Slack FJ: RAS is regulated by the let-7 microRNA family. Cell 120 : 635-647, 2005.

12. Esau C, Kang X, Peralta E, Hanson E, Marcusson EG, Ravichandran LV, Sun Y, Koo S, Perera RJ, Jain R, Dean NM, Freier SM, Bennett CF, Lollo B and Griffey R: MicroRNA143 regulates adipocyte differentiation. J Biol Chem 279: 52361-52365, 2004

13. Sharma-Walia N, Krishnan HH, Naranatt PP, Zeng L, Smith MS and Chandran B: ERK1/2 and MEK1/2 induced by Kaposi's sarcoma-associated herpesvirus (human herpesvirus 8) early during infection of target cells are essential for expression of viral genes and for establishment of infection. J Virol 79: 10308-10329, 2005.

14. English JM, Pearson G, Baer R and Cobb MH: Identification of substrates and regulators of the mitogen-activated protein kinase ERK5 using chimeric protein kinases. J Biol Chem 273: 3854-3860, 1998.

15. Calin GA, Sevignani C, Dumitru CD, Hyslop T, Noch E, Yendamuri S, Shimizu M, Rattan S, Bullrich F, Negrini M and Croce CM: Human microRNA genes are frequently located at fragile sites and genomic regions involved in cancers. Proc Natl Acad Sci USA 101: 2999-3004, 2004.

16. Chen PY and Meister G: microRNA-guided posttranscriptional gene regulation. Biol Chem 386: 1205-1218, 2005.

17. Gregory RI and Shiekhattar R: MicroRNA biogenesis and cancer. Cancer Res 65: 3509-3512, 2005.

18. Karube Y, Tanaka H, Osada H, Tomida S, Tatematsu Y, Yanagisawa K, Yatabe Y, Takamizawa J, Miyoshi S, Mitsudomi T and Takahashi T: Reduced expression of Dicer associated with poor prognosis in lung cancer patients. Cancer Sci 96: 111-115, 2005.

19. Fazi F, Rosa A, Fatica A, Gelmett V, De Marchis ML, Nervi C and Bozzoni I: A minicircuitry comprised of microRNA-223 and transcription factors NFI-A and $\mathrm{C} / \mathrm{EBP} \alpha$ regulates human granulopoiesis. Cell 123: 819-831, 2005. 